\title{
Adenosine antagonists for prevention of contrast-induced nephropathy: A meta-analysis of randomized controlled trials with trial sequential analysis
}

\author{
HONGBIN ZANG $^{1}$, QIONGYU ZHANG ${ }^{2}$ and XIAODONG LI ${ }^{1}$ \\ Departments of ${ }^{1}$ Cardiology and ${ }^{2}$ Neurology, Shengjing Hospital of China Medical University, Shenyang, \\ Liaoning 110004, P.R. China
}

Received October 7, 2018; Accepted March 1, 2019

DOI: $10.3892 /$ etm.2019.7566

\begin{abstract}
Contrast-induced nephropathy (CIN) is caused by intravascular administration of contrast agent. The efficacy of adenosine antagonists (AAs) in preventing CIN remains controversial, and its elucidation was the objective of the present meta-analysis. A trial sequential analysis (TSA) to assess the reliability of the pooled results was also performed. The Medline, Embase, Web of Science and Cochrane databases were searched to retrieve all published randomized controlled trials (RCTs) comparing AAs with controls in preventing CIN. Heterogeneity, publication bias and quality of studies were assessed. Sensitivity, cumulative and subgroup analyses were also performed. The risk of random errors was evaluated by TSA. A total of 17 trials with 1,483 subjects were included. Pooled results indicated that AAs significantly reduced the incidence of CIN [risk ratio, $0.53 ; 95 \%$ confidence interval (CI), 0.29-0.95; $\mathrm{P}=0.034]$ and the serum creatinine $(\mathrm{SCr})$ level after contrast media (CM) administration (standardized mean difference, $-0.24 ; 95 \% \mathrm{CI},-0.44$ to $-0.04 ; \mathrm{P}=0.019$ ). Meta-regression did not identify any significant source of heterogeneity. In the subgroup analyses, AAs tended to exhibit a greater prevention efficacy in trials with sample sizes of $\geq 70$, baseline $\mathrm{SCr}$ of $<1.5 \mathrm{mg} / \mathrm{dl}$ and low study quality. TSA on the incidence of CIN indicated that the required information size determined as $n=1,778$ was not reached, and that the cumulative Z-curve did not cross the TSA boundary. In conclusion,
\end{abstract}

Correspondence to: Professor Xiaodong Li, Department of Cardiology, Shengjing Hospital of China Medical University, 36 Sanhao Street, Shenyang, Liaoning 110004, P.R. China

E-mail: licardio@163.com

Abbreviations: CIN, contrast-induced nephropathy; AA, adenosine antagonist; TSA, trial sequential analysis; RCT, randomized controlled trial; SCr, serum creatinine; CM, contrast media; GFR, glomerular filtration rate; RIS, required information size; RR, risk ratio; CI, confidence interval; SMD, standardized mean difference

Key words: contrast-induced nephropathy, adenosine antagonists, theophylline, aminophylline, trial sequential analysis the present meta-analysis of data from current RCTs suggested that AAs reduce the incidence of CIN and the SCr levels after CM administration. However, TSA showed that the risk of having a false-positive result was greater than $5 \%$ in the meta-analysis of the incidence of CIN, indicating that more evidence is required to ensure the benefit of AAs in preventing CIN.

\section{Introduction}

With the development of diagnostic and interventional technology, the number of patients in whom contrast media (CM) are administered is continuously increasing. Those patients receiving intravascular $\mathrm{CM}$ administration are at risk of contrast-induced nephropathy (CIN). As one of the most common types of hospital-acquired kidney injury (accounting for $11-12 \%$ of all cases) (1), CIN is usually defined as an increase in serum creatinine ( $\mathrm{SCr}$ ) levels by $\geq 0.5 \mathrm{mg} / \mathrm{dl}$ or $\geq 25 \%$ within 48 or $72 \mathrm{~h}$ following CM exposure (2). Numerous factors may affect the risk of CIN, including age, hypertension, anemia, DM, atrial fibrillation, pre-existing renal dysfunction, insufficient circulation volume, type and volume of contrast agents and concomitant administration of nephrotoxic agents (3-6).

CIN may lead to dialysis, prolonged hospitalization and even increased mortality $(7,8)$. Effective prevention strategies are urgently required in clinics. By now, peri-procedural hydration with saline, administration of only the minimum required volume of CM, usage of low- or iso-osmolar CM and removal of nephrotoxic drugs have been widely accepted as methods to prevent CIN (9-11). Among the preventive medicines, adenosine antagonists (AAs) are of great interest. Animal studies have demonstrated that AAs increase renal blood flow and the glomerular filtration rate (GFR) after CM exposure by interrupting adenosine-induced vasoconstriction (12). However, several randomized controlled trials (RCTs) and meta-analyses have not consistently indicated a benefit of AAs in CIN prevention (13-15). Among those meta-analyses, only the most recent one suggested that AAs reduce the incidence of CIN (15). However, when a traditional meta-analysis is updated with new trials, the risk of a false-positive or false-negative result increases due to repeated significance testing $(16,17)$. To deal with this problem, trial sequential 
analysis (TSA) was recommended by the Copenhagen trial unit. As an interim analysis in a single trial, TSA may provide an information size calculation to ensure the reliability of the pooled estimate $(18,19)$. Furthermore, in TSA, the monitoring boundaries adjusted by random errors may detect the possibility of a false-positive or false-negative result before the required information size (RIS) is achieved $(20,21)$.

To determine the effect of AA in preventing CIN, an updated meta-analysis with inclusion of several recently published RCTs was performed. Furthermore, given the small sample sizes of the published trials and the dangers of overestimating the efficacy with traditional meta-analyses, TSA was also performed to assess the reliability of the pooled estimates.

\section{Materials and methods}

Search strategy. The literature search was performed using the Medline, Embase, Web of Science and Cochrane databases to retrieve all RCTs on AAs in preventing CIN published until December 31, 2017 by two investigators (HZ and QZ). The search terms were 'adenosine antagonists', 'theophylline', 'aminophylline', 'contrast-induced nephropathy', 'contrast-induced nephrotoxicity', 'contrast-medium nephrotoxicity', 'contrast medium-induced nephropathy', 'contrast-induced acute kidney injury', 'contrast-associated acute kidney injury' and 'contrast-associated nephropathy'. Trials were limited to those with human subjects only. A manual search was then performed (by $\mathrm{HZ}$ and QZ) on the results provided by the aforementioned databases. Before retrieving the full texts, the authors of the current study reviewed the titles and abstracts first. Full texts, rather than abstracts or meeting proceedings, were included. The references of the articles retrieved were also reviewed to identify potential trials for inclusion.

Selection criteria. Studies that met the following criteria were included: RCTs referring to CIN prevention; the intervention was AAs vs. control; aside from AA usage, there was no different intervention between the two arms in each trial; incidence of CIN must be reported.

Data extraction and quality assessment. Two investigators (HZ and QZ) separately extracted data from all of the primary studies meeting the selection criteria. The following information of each study was assembled: Sample size, rate of diabetes mellitus (DM), type and dose of CM, baseline renal function, outcome measures, protocols for the treatment with adenosine receptor antagonists and hydration protocols. The primary outcome measure was the development of CIN, defined as an increase in the SCr level by $\geq 0.5 \mathrm{mg} / \mathrm{dl}$ or $\geq 25 \%$ within 48-72 $\mathrm{h}$ following CM exposure (2). The secondary outcome measure was the SCr level after CM administration. Items that were used to assess the quality of studies included methods of randomization, methods of blinding, placebo control, reporting of losses to follow-up and reasons for losses to follow-up. The overall quality of studies was evaluated by determining the Jadad scores (22). In the case of any disagreement on the extracted data, a third reviewer (XL) would adjudicate.
Meta-analysis. Effect sizes for dichotomous data (incidence of $\mathrm{CIN}$ ) were presented as risk ratios (RRs) and 95\% confidence intervals (CIs), and for continuous data (SCr levels after CM administration), effect sizes were presented as the standardized mean differences (SMDs) and 95\% CIs. Heterogeneity was reported using $\mathrm{I}^{2}$ statistics, with values of $0-30 \%, 31-50 \%$ and $>50 \%$ representing low, moderate and significant heterogeneity, respectively. For low heterogeneity, a fixed-effects model was used; otherwise, a random-effects model was used. The meta-analysis was performed using STATA version 12.0 (StataCorp LP, College Station, TX, USA).

Publication bias and sensitivity analysis. Begg's test and Egger's test were performed to assess publication bias. A Begg's funnel plot was also generated. Sensitivity analysis was used to assess the effect of an individual study on the pooled estimate by removing one study at a time.

Meta-regression and subgroup analysis. Meta-regression was performed to identify the underlying sources of heterogeneity, which included sample sizes, volumes and osmotic pressures of CM, baseline renal function, proportion of patients with DM, type and dose of AAs, routes of AA administration, study quality and peri-procedural hydration protocols. Subgroup analyses were also performed according to sample size, osmotic pressure of $\mathrm{CM}$, baseline renal function and study quality.

Cumulative meta-analysis. Cumulative analysis was performed to observe the tendency of the pooled RRs for the incidence of CIN according to the publication year.

Trial sequential analysis. Meta-analyses are commonly updated when new evidence appears. However, repetitive testing of accumulating data runs the risk of producing random errors. To deal with this problem, TSA is used, in which the monitoring boundaries may avoid false-positive results and provide the benefit or futility of an intervention as early as possible. In the present study, the TSA of the incidence of CIN was performed using the TSA program version 0.9 beta (Www. ctu.dk/tsa) with $\alpha=5 \%$ and $1-\beta=80 \%$. The anticipated relative risk reduction was based on the pooled estimate of available trials. If the Z-curve crossed the conventional boundary of significance $(Z= \pm 1.96, P<0.05)$ but not the trial sequential significance boundary, the pooled estimate was considered to be at risk of false-positive results. Correspondingly, if the Z-curve neither crossed the conventional boundary of significance nor the trial sequential futility boundary, the pooled estimate was considered to be at risk of false-negative results. However, when the Z-curve crossed the RIS or the monitoring boundaries before the RIS is achieved, the pooled estimate was considered to be sufficiently reliable to make a firm conclusion (20).

\section{Results}

Identification of studies. From the initial literature search, 699 potentially relevant articles were identified. After the titles and abstracts of those articles were reviewed independently, 24 articles were regarded to be potentially 
qualified for inclusion. The full texts of those articles were searched. Subsequently, seven trials were excluded from the meta-analysis for various reasons (e.g., non-RCT studies, no reporting of CIN incidence, letters). Finally, 17 RCTs were identified and analyzed. The selection process of the present meta-analysis is presented in Fig. 1.

Features of studies included. The baseline characteristics of the 17 studies included are presented in Table I. These RCTs included 1,483 patients in total. The number of participants in each trial ranged from 30 to 280 (23-39). The percentage of patients with DM ranged from 0 to $100 \%$. A total of 3 studies used aminophylline $(24,33,36)$ and the others used theophylline $(23,25,32,34,35,37-39)$. Most studies included used iso- or low-osmolar CM, except for one study that used high-osmolar CM (27). AAs were administered either intravenously or orally in those trials. In 9 studies, CIN was defined as an increase in $\mathrm{SCr}$ levels by $\geq 0.5 \mathrm{mg} / \mathrm{dl}$ from baseline $(25,26,28-30,32,33,36,38)$. In two studies, CIN was defined as a $\geq 25 \%$ increase in $\mathrm{SCr}$ levels from baseline $(24,27)$, and CIN was defined as either in five studies $(31,34,35,37,39)$. In most studies, there was no difference in the baseline $\mathrm{SCr}$ between the AA and control groups $(23,24,26-33,35-39)$. However, in two studies, the baseline SCr was unfavorable for the AA group $(25,34)$.

Quality assessment of trials. Of the trials included, 4 exhibited high quality (Jadad scores $\geq 3)(25,29,35,39)$. The other studies were identified as having low quality due to lack of randomization processes, methods for blinding or losses to follow-up (Jadad scores <3) (23,24,26-28,30-34,36-38). The overall quality of studies assessed by the Jadad scores is presented in Table II.

Publication bias. According to Begg's test $(\mathrm{P}=0.773)$ and Egger's test $(\mathrm{P}=0.760)$, there was no publication bias across the included trials. The funnel plot is presented in Fig. 2. Sensitivity analysis indicated that no single trial significantly influenced the pooled estimate.

Efficacy of AA to reduce the incidence of CIN. A total of 1,483 patients were included from 17 RCTs reporting data on the incidence of CIN, with 737 and 746 patients allocated to the AA and control groups, respectively. The overall incidence of CIN was $8.90 \%$. CIN occurred in 5.83 and $11.93 \%$ of the subjects in the AA and control groups, respectively. There was moderate heterogeneity across the studies $\left(\mathrm{I}^{2}=47.4 \%\right.$; $\mathrm{P}=0.016$ ) and therefore, a random-effects model was used for meta-analysis. The pooled result revealed that AAs significantly reduced the incidence of $\mathrm{CIN}(\mathrm{RR}=0.53 ; 95 \% \mathrm{CI}$, 0.29-0.95; P=0.034; Fig. 3).

Meta-regression revealed that the sample size ( $\geq 70$ vs. $<70$; $\mathrm{P}=0.180)$, volume ( $\geq 150$ vs. $<150 \mathrm{ml} ; \mathrm{P}=0.827)$ and osmotic pressures of $\mathrm{CM}(\mathrm{P}=0.708)$, baseline renal function ( $\geq 1.5$ vs. $<1.5 \mathrm{mg} / \mathrm{dl} ; \mathrm{P}=0.210$ ), proportion of subjects with DM ( $\geq 50$ vs. $<50 \%$; $\mathrm{P}=0.635$ ), type of AA (theophylline vs. aminophylline; $\mathrm{P}=0.672$ ) and dose of theophylline and aminophylline (>250 vs. $\leq 250 \mathrm{mg} ; \mathrm{P}=0.180$ ), route of AA administration (oral vs. intravenous; $\mathrm{P}=0.239$ ), study quality (Jadad scores, $\geq 3$ vs. $<3$; $\mathrm{P}=0.205$ ), and peri-procedural hydration protocols (with hydration vs. without hydration; $\mathrm{P}=0.936$ )

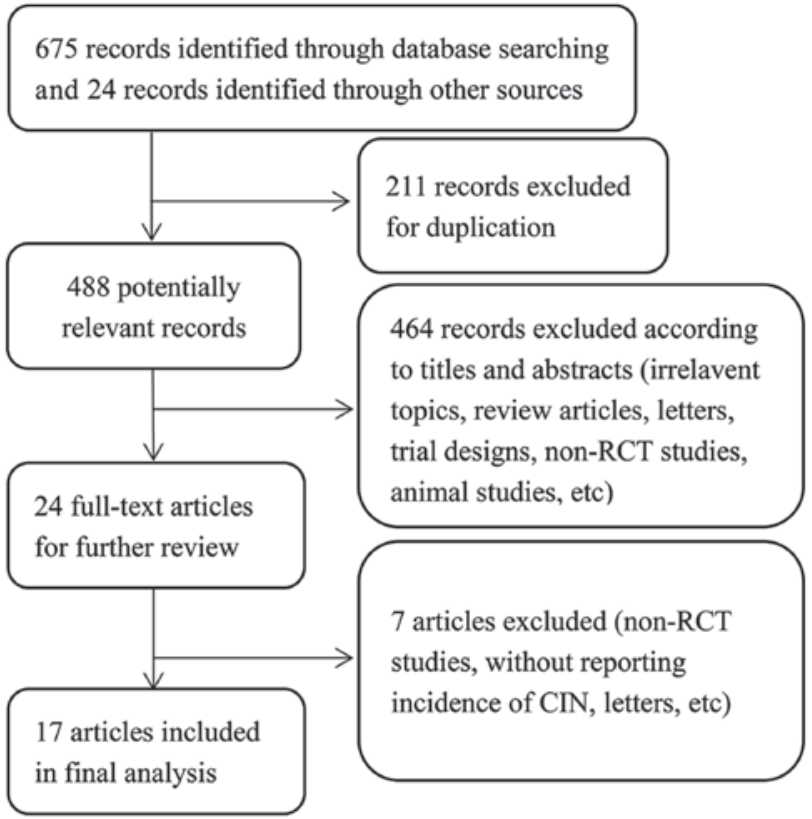

Figure 1. Flow chart illustrating the selection of studies for the present meta-analysis. RCT, randomized controlled trial; CIN, contrast-induced nephropathy.

did not explain for the heterogeneity of the CIN prevention effect of AAs across studies. Subgroup analyses indicated that AAs reduced the incidence of CIN in trials with a sample size of $\geq 70$ ( RR=0.34; 95\% CI, 0.14-0.81; $\mathrm{P}=0.015$; Fig. 4), baseline $\mathrm{SCr}<1.5 \mathrm{mg} / \mathrm{dl}(\mathrm{RR}=0.36 ; 95 \% \mathrm{CI}, 0.14-0.89 ; \mathrm{P}=0.028 ;$ Fig. 5$)$, and of low quality $(\mathrm{RR}=0.41 ; 95 \% \mathrm{CI}, 0.21-0.83 ; \mathrm{P}=0.013$; Fig. 6), but not in trials with sample sizes $<70(\mathrm{RR}=0.81$; 95\% CI, 0.38-1.71; $\mathrm{P}=0.581$; Fig. 4), baseline $\mathrm{SCr} \geq 1.5 \mathrm{mg} / \mathrm{dl}$ (RR=0.76; 95\% CI, 0.38-1.51; P=0.435; Fig. 5) and of high quality ( $R R=1.07 ; 95 \%$ CI, 0.38-3.02; $\mathrm{P}=0.901$; Fig. 6), which may in part explain for the underlying source of heterogeneity. When trials were grouped by osmotic pressure of CM, AAs did not significantly reduce the incidence of CIN neither in trials using low-osmolar CM ( $\mathrm{RR}=0.62 ; 95 \% \mathrm{CI}, 0.32-1.20$; $\mathrm{P}=0.159$; Fig. 7) nor in trials using iso-osmolar $\mathrm{CM}(\mathrm{RR}=1.03$; 95\% CI, 0.06-17.07; P=0.981; Fig. 7). However, since only one trial used high-osmolar CM (27), no pooled analysis was possible, although the trial did indicate a significant decrease in the incidence of CIN by theophylline. Furthermore, in another trial, patients receiving either or low- and iso-osmolar CM were assessed (34). This trial did not specify which of the patients were treated by low- and iso-osmolar CM. Thus, in this trial, although administration of theophylline significantly reduces the incidence of $\mathrm{CIN}$, the effects of AAs on the CIN induced by different osmotic pressures of CM could not be analyzed separately.

As the studies were added one by one, the cumulative meta-analysis indicated that the value of the pooled RRs was unstable and the corresponding 95\% CIs did not become narrower, at times even with a $\mathrm{P}>0.05$ (Fig. 8).

In the TSA, the anticipated relative risk reduction, which was based on the pooled estimate of available trials, was $47 \%$ for the incidence of CIN. The RIS of 1,778 was not reached. In addition, the cumulative $\mathrm{Z}$-curve crossed the conventional boundary for benefit $(\mathrm{P}=0.05)$ but not the trial sequential 


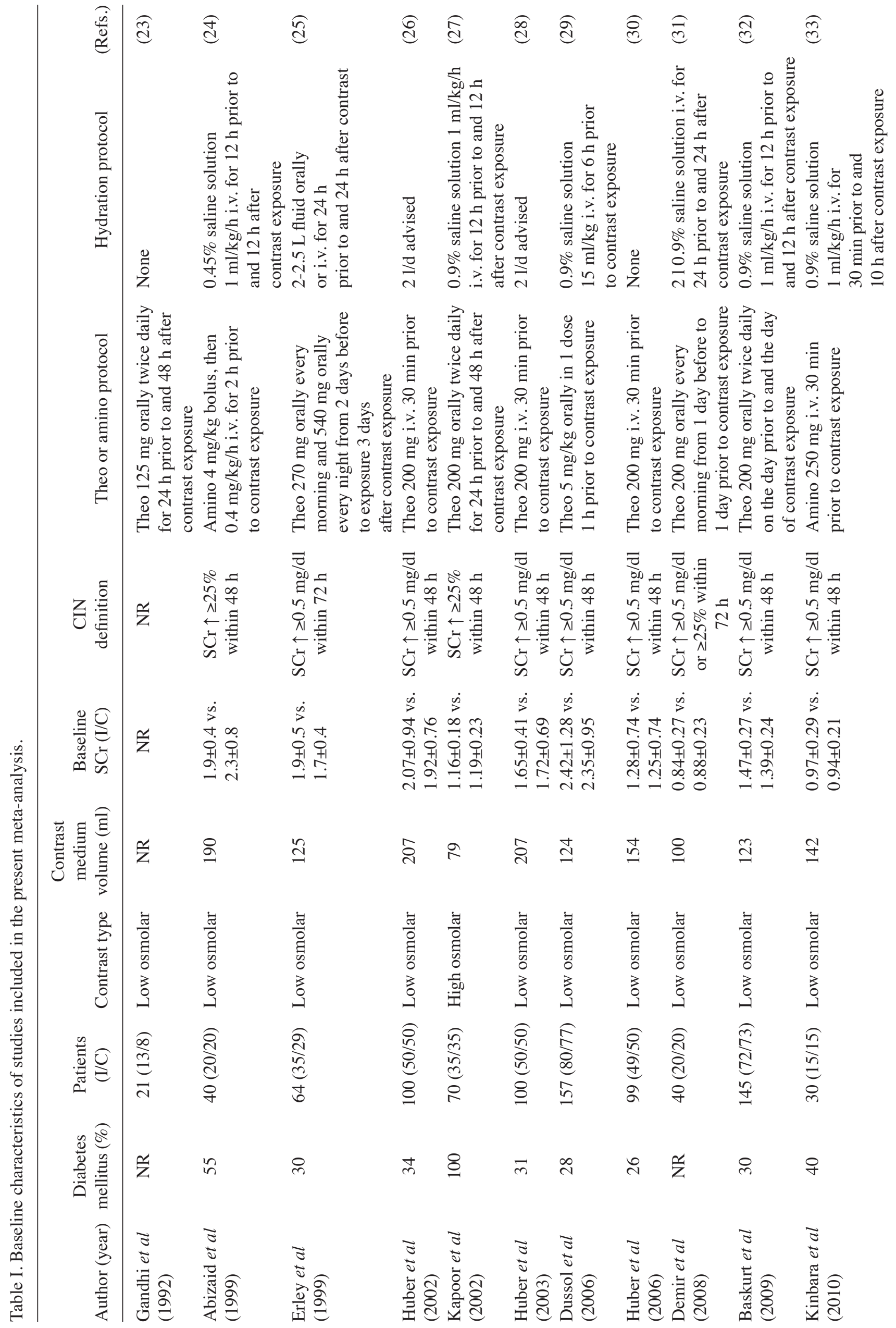




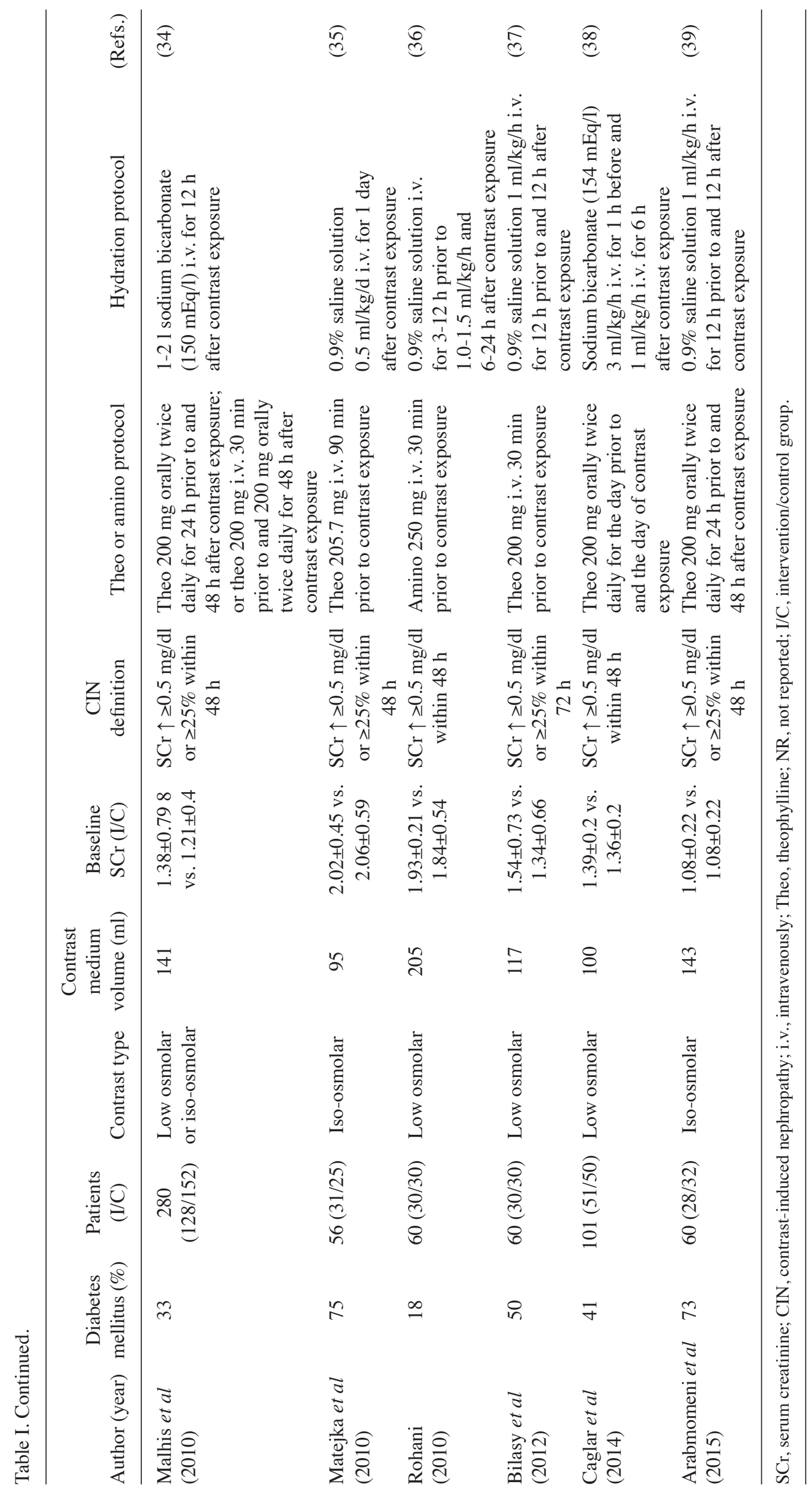




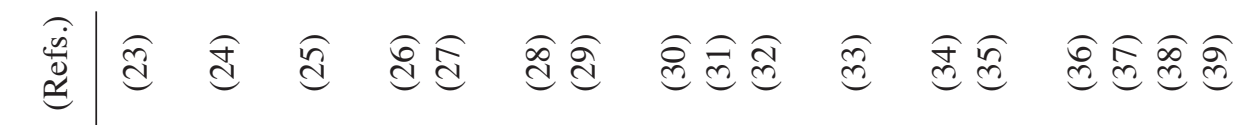

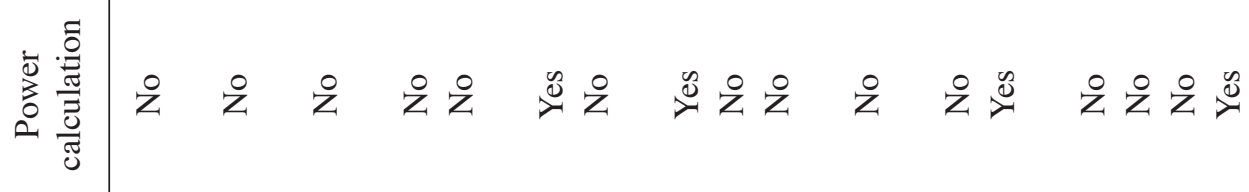

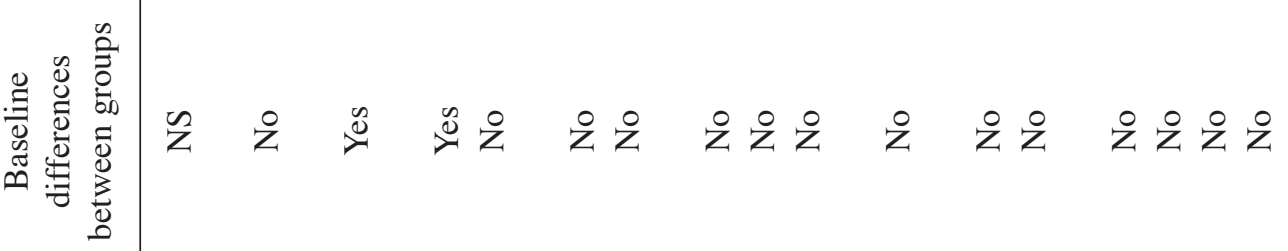

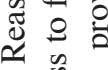
응

究争

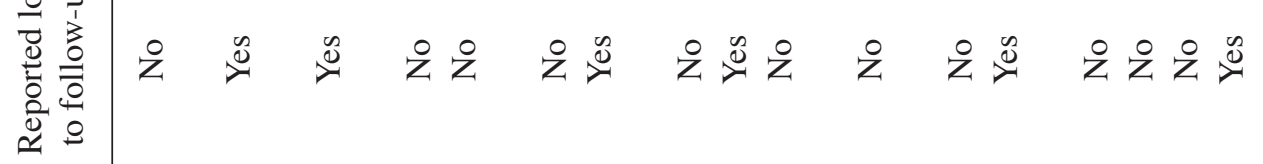

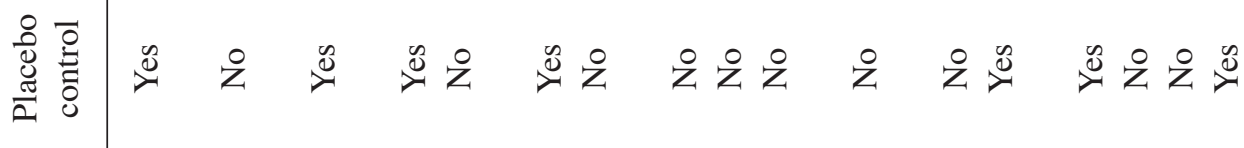

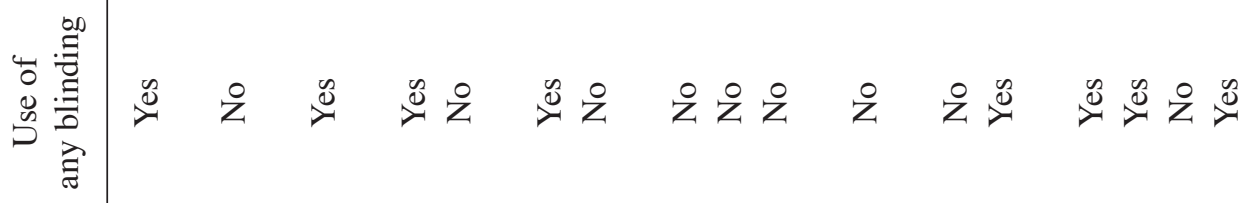
:

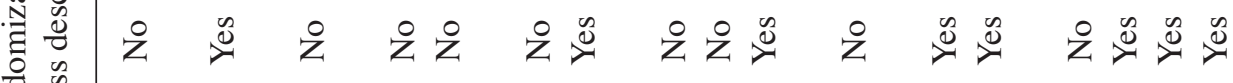

喜

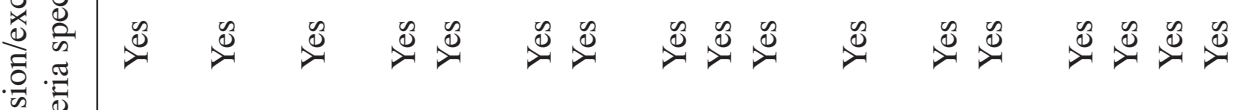

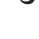

总

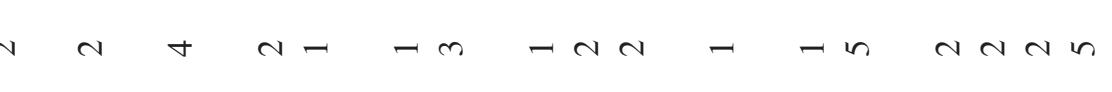




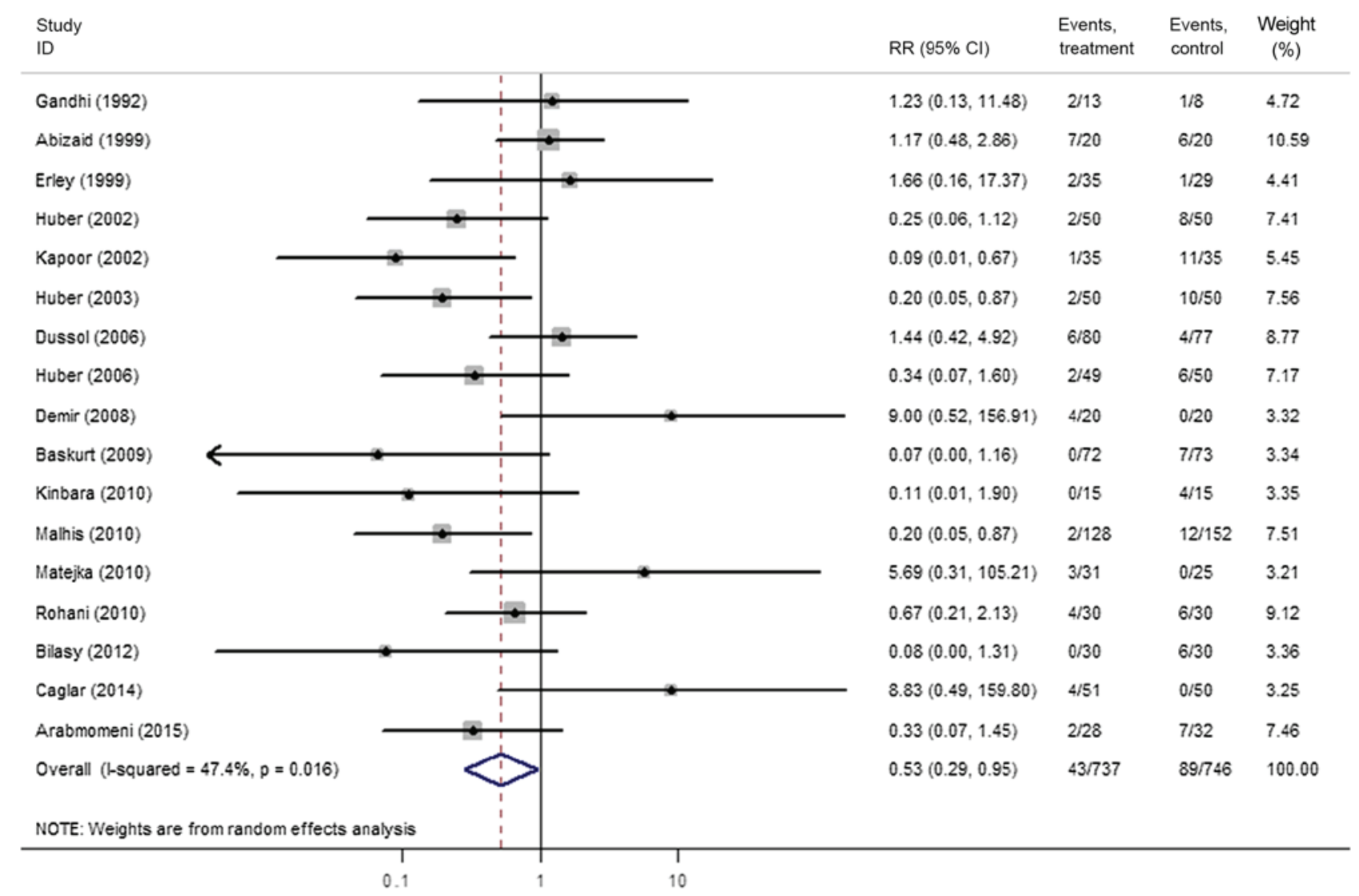

Figure 2. Funnel plot for assessment of bias of studies on contrast-induced nephropathy with pseudo-95\% confidence limits. RR, risk ratio.

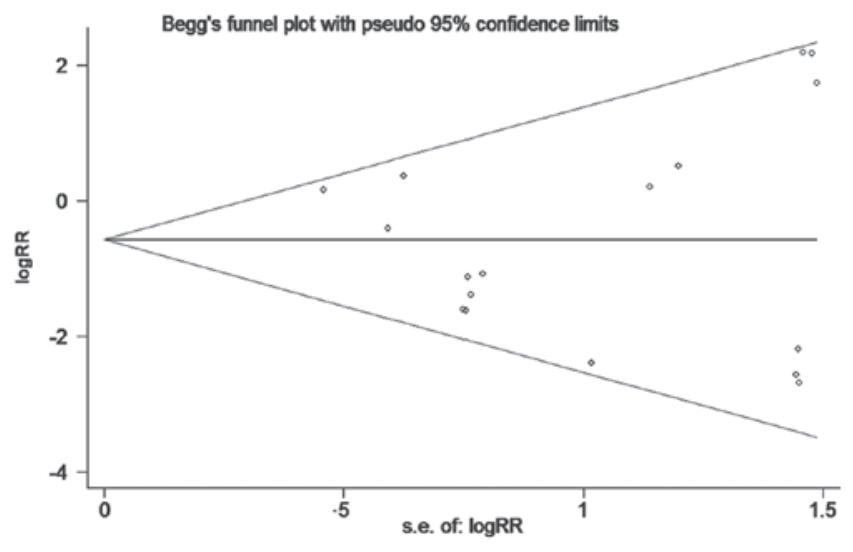

Figure 3. Forest plot of RR for the incidence of contrast-induced nephropathy. The diamond indicates the pooled RR and its 95\% CI. The horizontal lines indicate the $95 \%$ CI of RRs in each trail. The squares indicate RRs in each trail and the weight of each trial. RR, risk ratio; $\mathrm{CI}$, confidence interval.

monitoring boundary for benefit, indicating a $>5 \%$ false-positive risk for the result of a $47 \%$ reduction in the incidence of CIN with AAs vs. control (Fig. 9). TSA was also exclusively performed in high-quality studies. The RIS was not reached and the Z-curve did not cross the conventional or trial sequential significance boundary (Fig. 10).

SCr level after CM administration. Among the studies included in the present meta-analysis, three trials did not contain data on the SCr level after CM administration. A total of 12 trials reported the $\mathrm{SCr}$ levels at $48 \mathrm{~h}$ after $\mathrm{CM}$ administration. Only two trials reported on the $\mathrm{SCr}$ levels at $72 \mathrm{~h}$ after CM administration. Thus, a pooled analysis on the SCr levels at $48 \mathrm{~h}$ after $\mathrm{CM}$ administration was performed, revealing and a significant reduction in the AA vs. control group (SMD, -0.24 mg/dl; $95 \%$ CI, -0.44 to $-0.04 ; \mathrm{P}=0.019 ; \mathrm{I}^{2}=62.3 \%$; Fig. 11$)$.

Dialysis and mortality. A total of 10 trials reported on the cases that required dialysis after $\mathrm{CM}$ administration. Out of 501 patients in the AA group, $3(0.60 \%)$ required dialysis, while none of the 517 patients in the control group required dialysis. In-hospital mortality was reported by only four trials. Mortality occurred in $1(0.69 \%)$ of 145 patients in the AA group and $3(2.07 \%)$ of 145 patients in the control group. With those low dialysis and mortality rates, it was impossible to perform meta-analyses on these two outcome measures.

\section{Discussion}

The present study included 17 RCTs, and of 1,483 the participants, 132 experienced CIN [AA group: 43/737 (5.83\%); control group: 89/746 (11.93\%)]. The meta-analysis suggested that AAs significantly reduced the risk of CIN and the $\mathrm{SCr}$ level after CM exposure. In addition, TSA indicated that the trials included failed to provide firm evidence for a $47 \%$ relative risk reduction in the incidence of CIN.

The pathophysiological mechanism of CIN remain incompletely understood. It is widely accepted that the direct toxicity of CM, reduced renal blood flow caused by vasoconstrictors and oxidative stress caused by reactive 


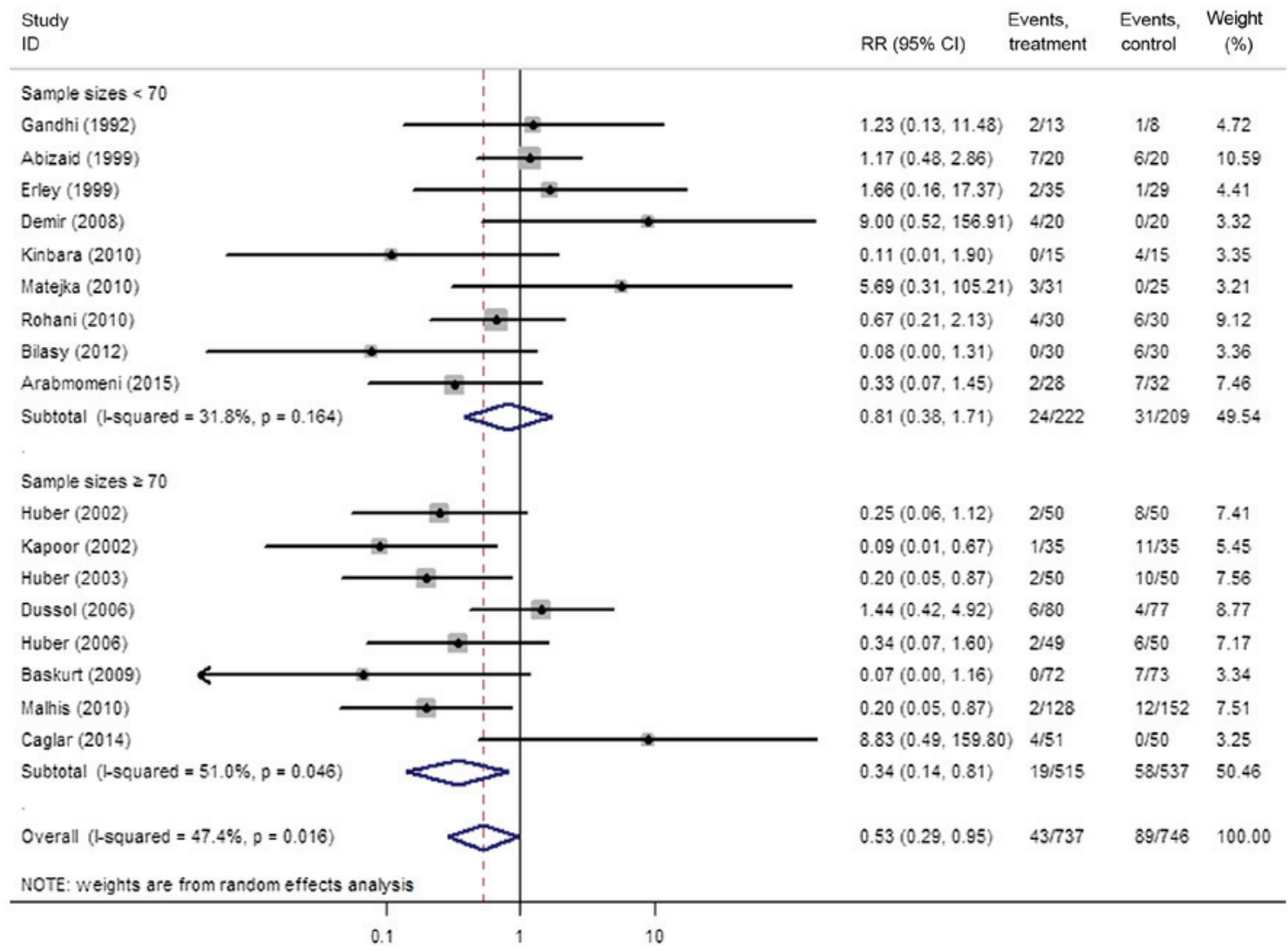

Figure 4. Subgroup analysis according to sample sizes. The diamonds indicate the pooled RRs and their 95\% CI. The horizontal lines indicate the $95 \%$ CI of RRs in each trail. The squares indicate RRs in each trail and the weight of each trial. RR, risk ratio; CI, confidence interval.

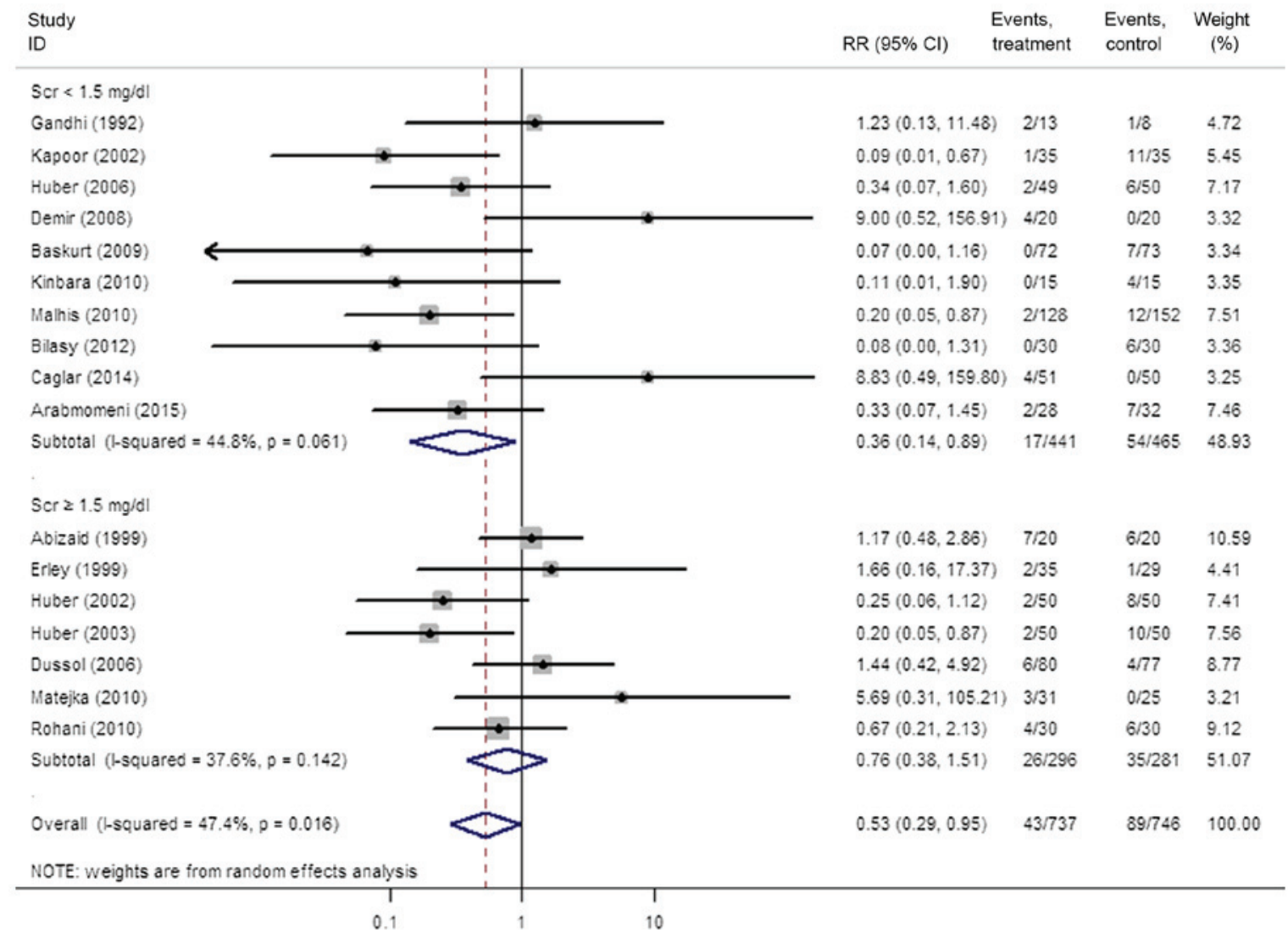

Figure 5. Subgroup analysis according to baseline renal function. The diamonds indicate the pooled RRs and their 95\% CI. The horizontal lines indicate the $95 \%$ CI of RRs in each trail. The squares indicate RRs in each trail and the weight of each trial. SCr, serum creatinine; RR, risk ratio; CI, confidence interval. 


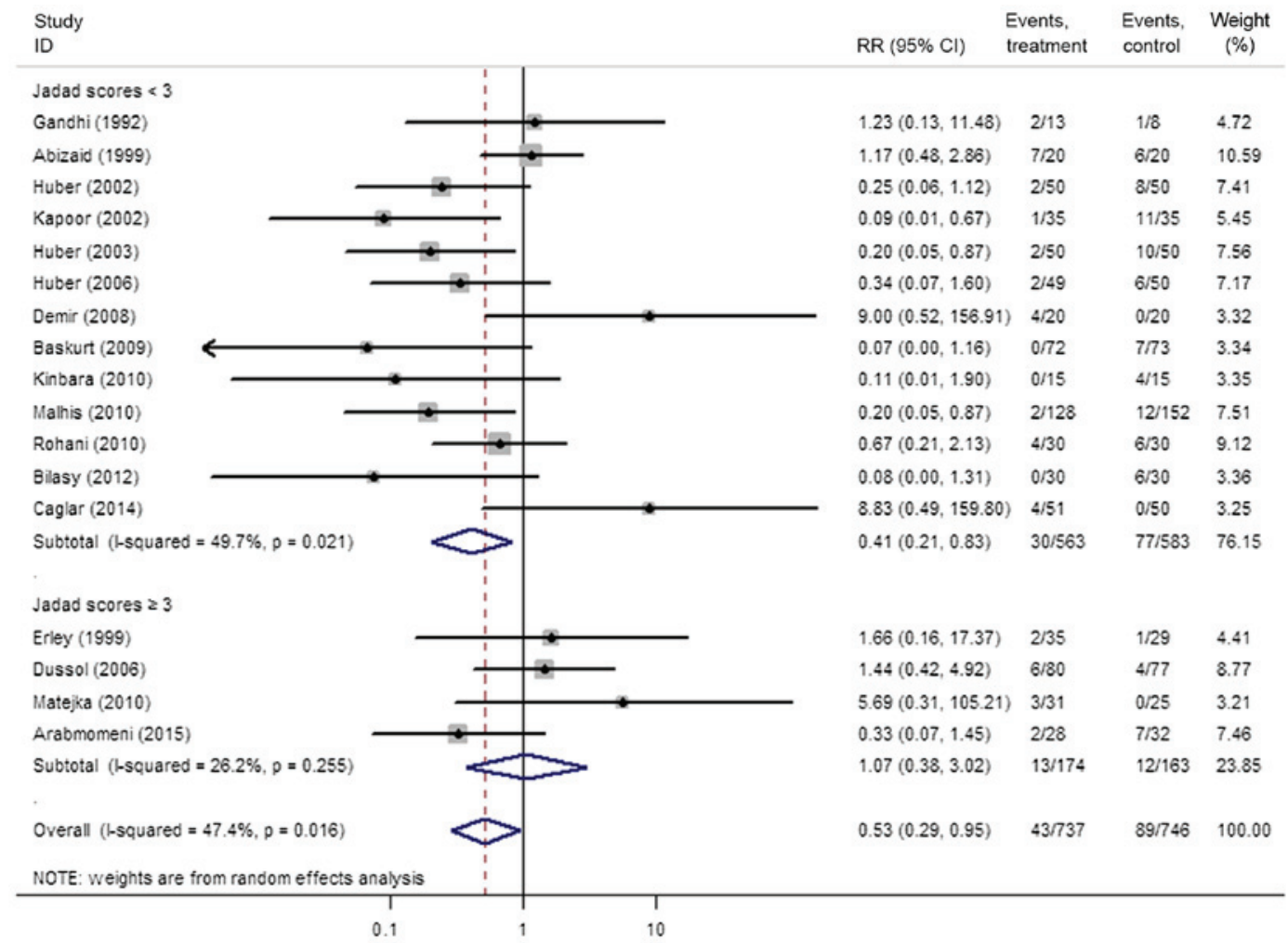

Figure 6. Subgroup analysis according to study quality. The diamonds indicate the pooled RRs and their 95\% CI. The horizontal lines indicate the $95 \%$ CI of RRs in each trail. The squares indicate RRs in each trail and the weight of each trial. RR, risk ratio; CI, confidence interval.

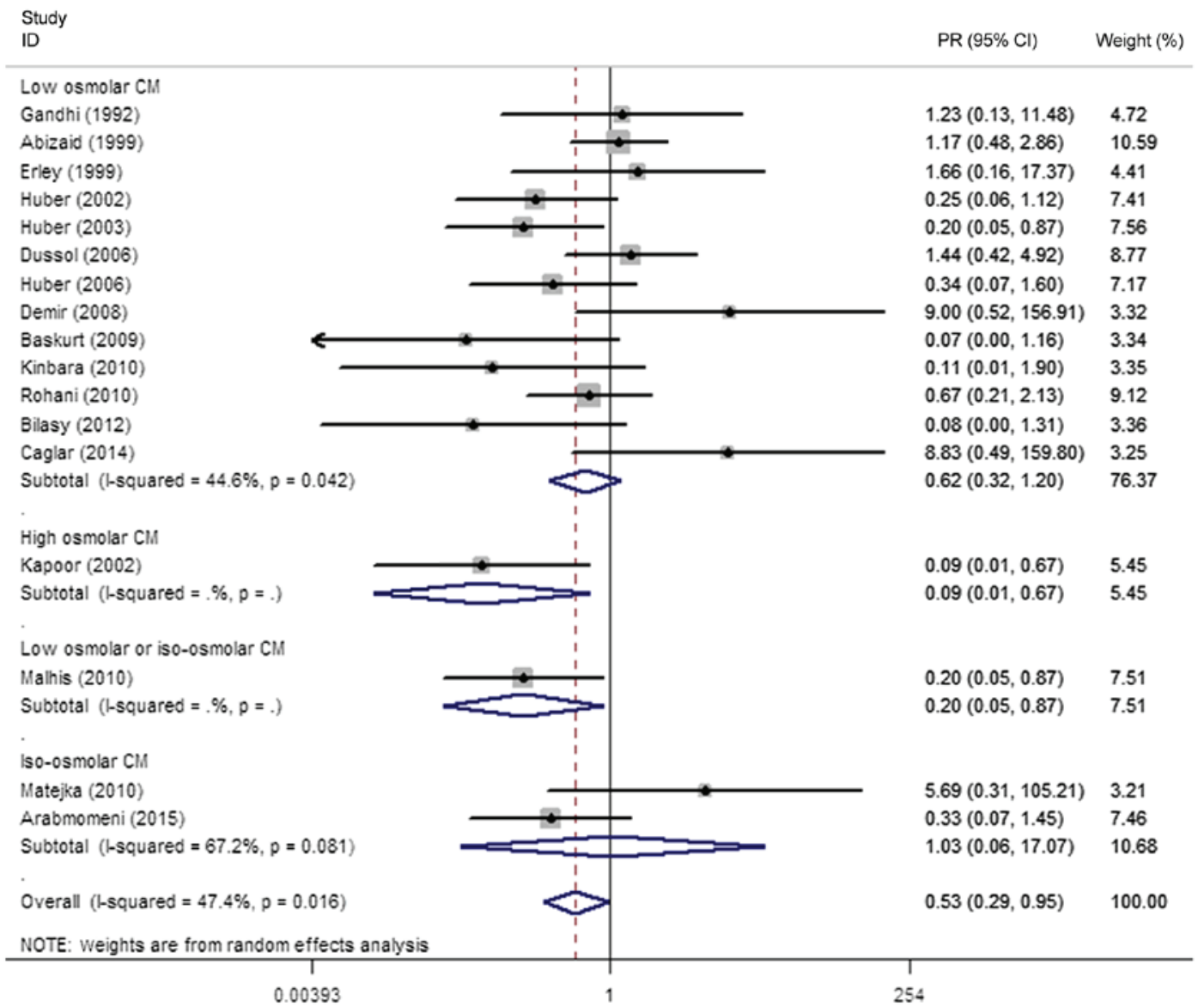

Figure 7. Subgroup analysis according to osmotic pressure of contrast media. The diamonds indicate the pooled RRs and their 95\% CI. The horizontal lines indicate the $95 \%$ CI of RRs in each trail. The squares indicate RRs in each trail and the weight of each trial. CM, contrast media; RR, risk ratio; CI, confidence interval. 


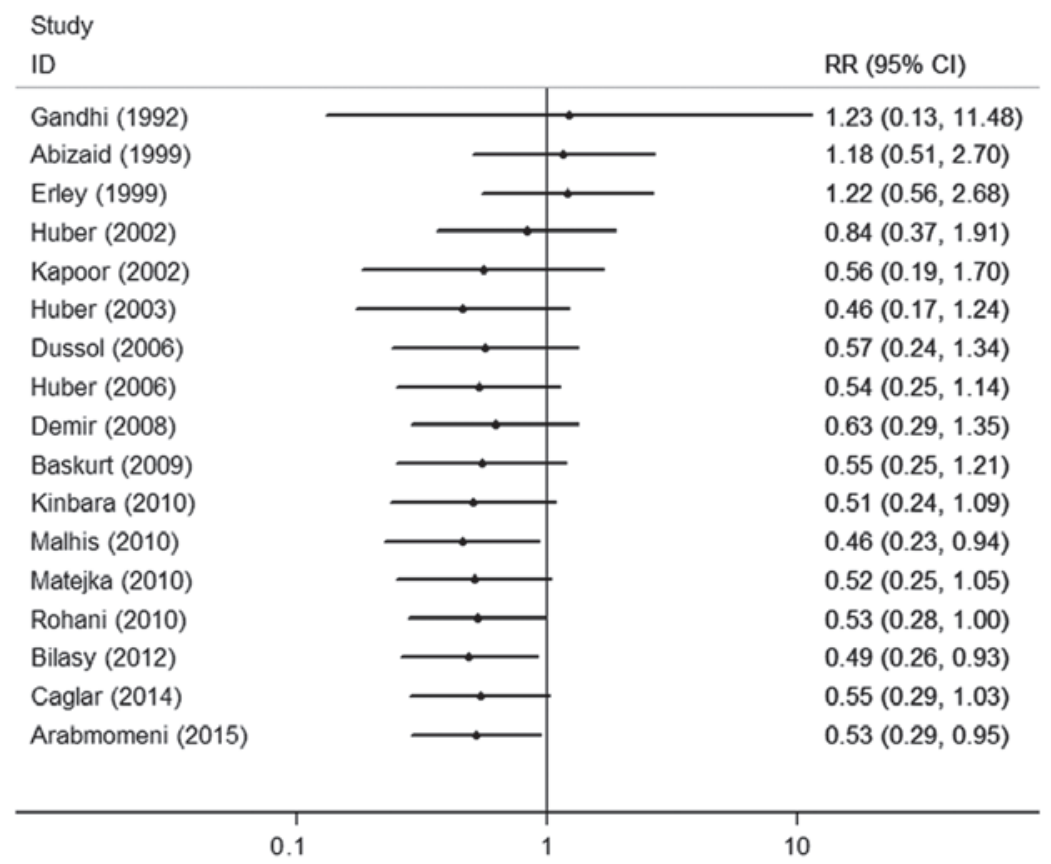

Figure 8. Cumulative meta-analysis of the incidence of contrast-induced nephropathy. The horizontal lines indicate the 95\% CI of the pooled RRs until the published year. RR, risk ratio; CI, confidence interval.

oxygen species are mainly responsible for the development of CIN. The direct toxicity of CM toward renal tubule cells is the primary factor. When irritated by $\mathrm{CM}$, renal tubule cells release various vasoconstrictors and generate reactive oxygen species that induce programmed cell death $(40,41)$. Adenosine is the most important vasoconstrictor involved in CIN, which causes vasoconstriction of the vas afferens by activating adenosine 1 receptor (42). Thus, by blocking adenosine 1 receptor, AAs increase renal blood flow and the GFR (43).

Several clinical studies have investigated AAs for preventing CIN. However, contradictory results were obtained. Due to the small sample sizes of those studies, meta-analyses were performed to determine the protective effect of AAs. There have been three previous meta-analyses in this field. The meta-analysis of seven RCTs by Ix et al (13) indicated that AA administration caused a decrease in the $\mathrm{SCr}$ levels after $\mathrm{CM}$ exposure (SMD $=-0.13 \mathrm{mg} / \mathrm{dl} ; 95 \% \mathrm{CI}$, -0.22 to -0.06 ), but they did not assess the incidence of CIN as an outcome. A later meta-analysis including nine RCTs by Bagshaw and Ghali (14) reported that AAs did not reduce the incidence of CIN (odds ratio, 0.40; 95\% CI, 0.14-1.16) but improved renal function after $\mathrm{CM}$ exposure compared with the controls ( $\mathrm{SCr}, \mathrm{SMD}=-0.17 \mathrm{mg} / \mathrm{dl} ; 95 \% \mathrm{CI},-0.28$ to -0.06 ). The most recent meta-analysis of 13 RCTs by Dai et al (15) indicated that AAs not only improved renal function after $\mathrm{CM}$ exposure $(\mathrm{SCr}, \mathrm{SMD}=-0.31 \mathrm{mg} / \mathrm{dl} ; 95 \% \mathrm{CI},-0.50$ to -0.11) but also reduced the incidence of CIN ( $R R=0.48$; 95\% CI, 0.26-0.89). However, as meta-analyses are updated, the false-positive risk increases. In meta-analyses, a single significance test can be considered reliable once the required information size is surpassed $(20,21)$. However, meta-analyses are often performed before required information sizes are reached and are commonly updated when new trials are published. When meta-analyses are updated (before required information sizes are reached), they are repeatedly subjected to the significance testing over time. The repeated significance testing on accumulating data is known to inflate the overall false-positive risk. Simulation studies suggest that if repeated significance testing is done in meta-analyses and $\mathrm{P}<0.05$ was considered to indicate a statistical significant difference, then the actual false-positive risk will be between 10 and $30 \%$ (17). This phenomenon is commonly known as 'multiplicity due to repeated significance testing' (17). TSA may help minimize the risk of a false-positive or false-negative result in a meta-analysis by RISs and monitoring boundaries. In the present study, the TSA on the incidence of CIN indicated that the Z-curve neither crossed the trial sequential significance boundary nor reached RIS, indicating that it was not possible to draw a reliable conclusion of a $47 \%$ reduction in the incidence of CIN and another 295 subjects were required to reach the RIS. Some of the included studies were indicated to be of low quality. To minimize the effects of these low-quality studies on the results of the meta-analysis, TSA was repeated with only high-quality studies included. This did not markedly alter the results of the analysis, and the results still implied that the benefit of AAs in CIN could not be reliably determined.

Using regression analysis, Dai et al (15) indicated that the baseline SCr and study quality partly explained for the heterogeneity in their meta-analysis. However, in the present study, no source of heterogeneity was detected by regression analysis. This may have been caused by the newly added articles. Compared with the meta-analysis by Dai et al (15), the present study included four other trials. The baseline $\mathrm{SCr}$ was $<1.5 \mathrm{mg} / \mathrm{dl}$ in all those trials. The sample sizes of those articles ranged from 40 to 101 and one of the studies was of high quality. Furthermore, the quality of trials in previous meta-analyses was reviewed. One study that was of high quality was originally evaluated as having low quality. 


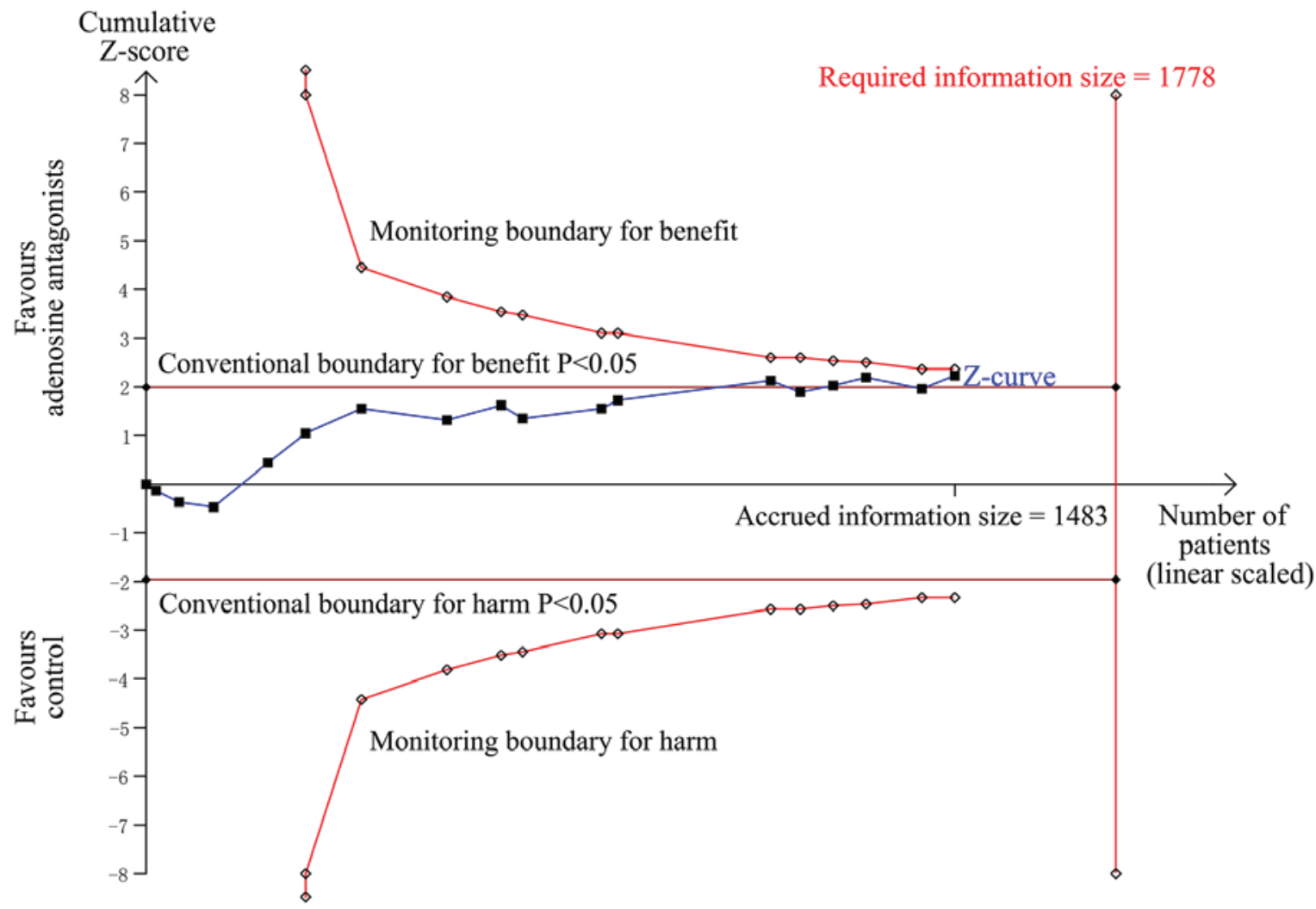

Figure 9. Trial sequential analysis of 17 trials on the incidence of CIN. The required information size was 1,778 , based on an anticipated intervention effect resembling a relative risk reduction of $47 \%$. The control event proportion was estimated from the incidence of CIN in the control group and a diversity of $54 \%, \alpha=5 \%$ and $1-\beta=80 \%$. The required information size was not reached and the $\mathrm{Z}$-curve crossed only the conventional boundary $(\mathrm{P}<0.05)$ but not the trial sequential monitoring boundary for benefit, suggesting the possibility of a false-positive result. CIN, contrast-induced nephropathy.

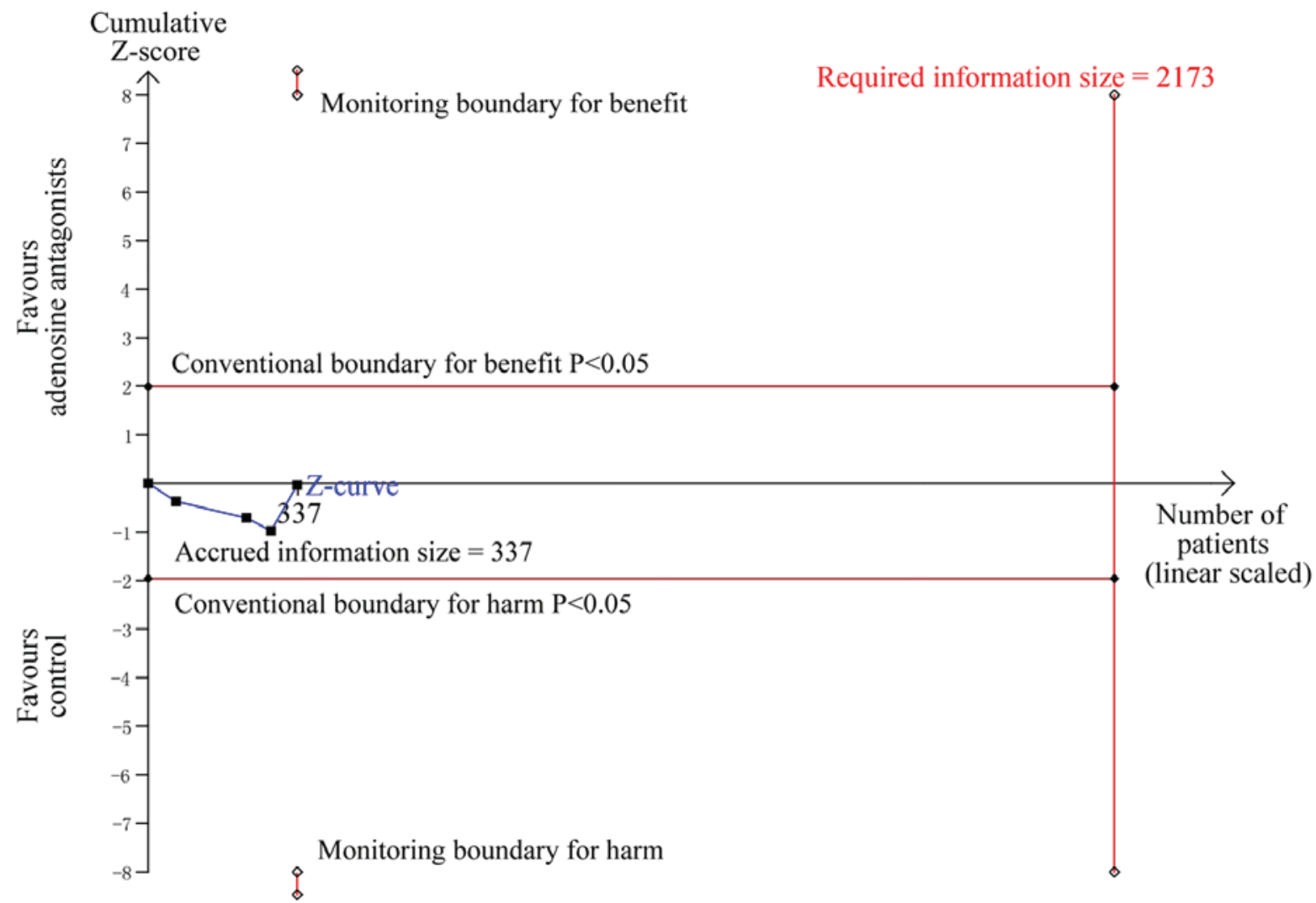

Figure 10. Trial sequential analysis of four high-quality trials on the incidence of CIN. The required information size was 2,173, based on an anticipated intervention effect of $47 \%$ relative risk reduction. The control event proportion was estimated from the incidence of CIN in the control group and a diversity of $36 \%, \alpha=5 \%$, and $1-\beta=80 \%$. 


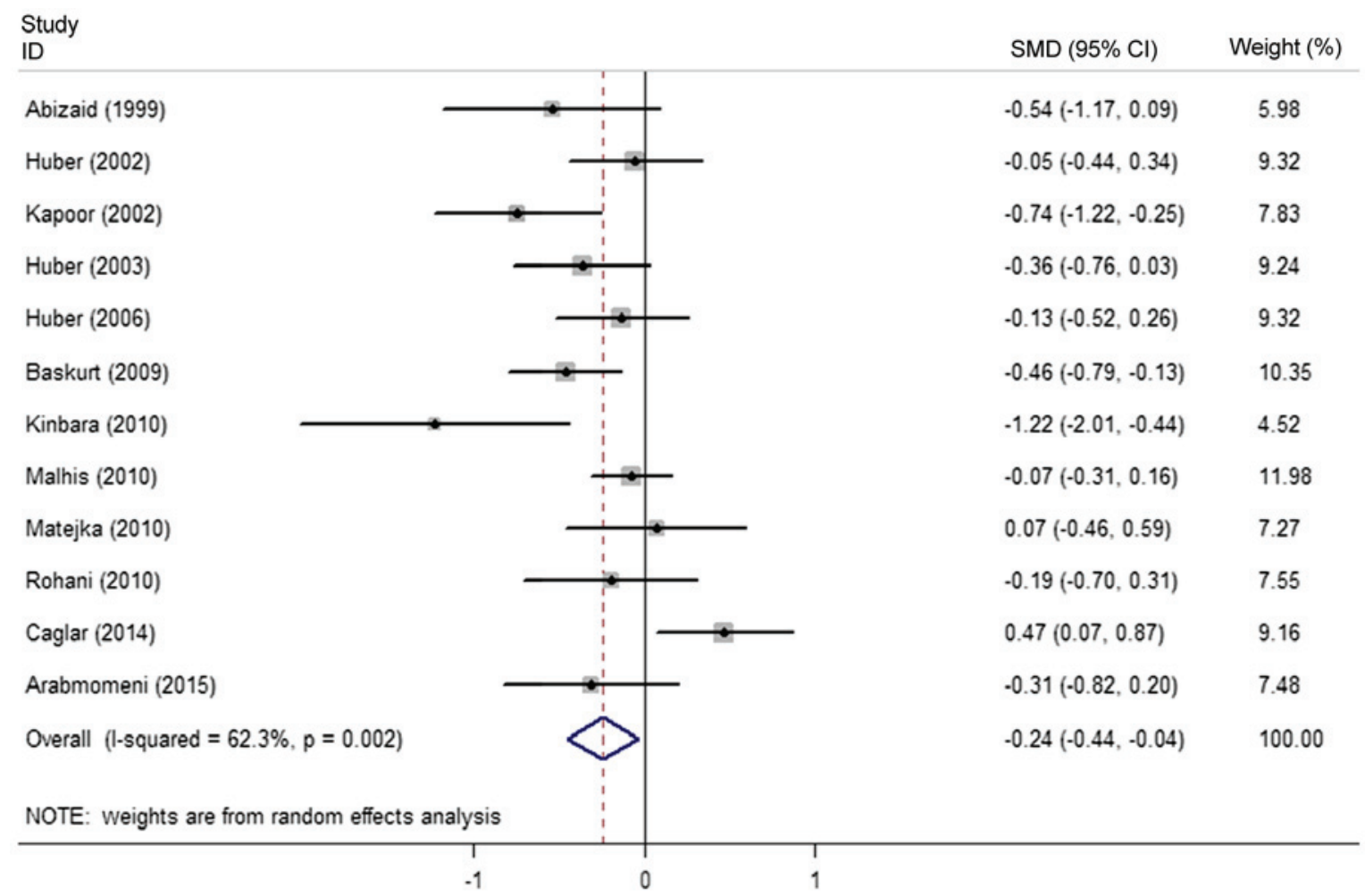

Figure 11. Forest plot of difference in the serum creatinine level at $48 \mathrm{~h}$ after contrast media administration between the adenosine antagonist and control groups. The diamond indicates the pooled SMD and its 95\% CI. The horizontal lines indicate the 95\% CI of SMDs in each trail. The squares indicate SMDs in each trail and the weight of each trial. SMD, standardized mean difference; CI, confidence interval.

Furthermore, although no source of heterogeneity was detected by regression analysis, subgroup analyses indicated that AAs tended to exhibit a greater prevention effect in trials with sample sizes of $\geq 70$, baseline $\mathrm{SCr}$ of $<1.5 \mathrm{mg} / \mathrm{dl}$ and low quality. However, the significant benefit in low-quality studies may be due to underlying bias. TSA revealed that 1,836 patients from high-quality studies were required to draw a firm conclusion.

Attention should be paid to the safety of AAs. AAs may induce adverse reactions and malignant arrhythmia is of particular concern. In the present meta-analysis, six articles including 658 subjects reported on the side effects of AAs $(26,29,30,35,38,39)$. Some of those subjects experienced coronary heart disease, heart failure and renal insufficiency, all of whom were at high risk for arrhythmia induced by AAs. However, there was only one case of side effects during the experimental period, namely of transient sinus tachycardia after AA administration (26), but no malignant arrhythmia occurred. Previous studies indicated that the side effects of AAs were likely to occur only with serum concentrations of $>20 \mathrm{~g} / \mathrm{ml}$ (44). Therefore, the dosage and speed of AA administration should be controlled, particularly when given intravenously. Slow infusion of $250 \mathrm{mg}$ theophylline or aminophylline over $30 \mathrm{~min}$ may be recommended.

Of note, the present study had several limitations. First, the limited number of high-quality studies may have limited the reliability and credibility of the pooled results. Furthermore, only few trials included in the present meta-analysis reported on the side effects of AAs. Therefore, no pooled results were available to assess the safety of AAs, which may induce arrhythmias in patients with coronary heart disease and heart failure. Finally, few trials were designed to assess the effect of AAs on mortality. Thus, the present meta-analysis did not provide any evidence regarding those important outcomes.

In conclusion, meta-analysis of the available data from the RCTs indicated a significant reduction in the incidence of CIN and the $\mathrm{SCr}$ levels with AAs vs. control for patients receiving $\mathrm{CM}$. However, TSA on the incidence of CIN detected the risk of a false-positive result, indicating that more evidence is required to ensure the benefit of AAs in preventing CIN. Future studies should be of high quality and investigate the effect of AAs on clinically relevant outcomes, including in-hospital morbidity, mortality and the requirement for dialysis.

\section{Acknowledgements}

Not applicable.

\section{Funding}

No funding was received.

\section{Availability of data and materials}

The datasets used or analyzed during the current study are available from the corresponding author on reasonable request.

\section{Authors' contributions}

HZ conceived and designed the study. HZ, QZ and XL performed the literature search and data extraction. HZ performed the statistical analysis. HZ and QZ wrote the 
manuscript. HZ, QZ and XL reviewed and edited the manuscript. All authors read and approved the final manuscript.

\section{Ethics approval and consent to participate}

Not applicable.

\section{Patient consent for publication}

Not applicable.

\section{Competing interests}

The authors declare that they have no competing interests.

\section{References}

1. Maliborski A, Zukowski P, Nowicki G and Bogusławska R: Contrast-induced nephropathy-a review of current literature and guidelines. Med Sci Monit 17: RA199-RA204, 2011.

2. McCullough PA: Contrast-induced acute kidney injury. J Am Coll Cardiol 51: 1419-1428, 2008.

3. Mehran R, Aymong ED, Nikolsky E, Lasic Z, Iakovou I, Fahy M, Mintz GS, Lansky AJ, Moses JW, Stone GW, et al: A simple risk score for prediction of contrast-induced nephropathy after percutaneous coronary intervention: Development and initial validation. J Am Coll Cardiol 44: 1393-1399, 2004.

4. Kumar S, Nair RK, Aggarwal N, Abbot AK, Muthukrishnan J and Kumar KV: Risk factors for contrast-induced nephropathy after coronary angiography. Saudi J Kidney Dis Transpl 28 : 318-324, 2017.

5. Valappil SP, Kunjukrishnapillai S, Iype M, Koshy AG, Viswanathan S, Gupta PN, Velayudhan RV and Ali FM: Predictors of contrast induced nephropathy and the applicability of the Mehran risk score in high risk patients undergoing coronary angioplasty-A study from a tertiary care center in South India. Indian Heart J 70: 399-404, 2018.

6. Cinar T, Keskin M and Kaya A: Atrial fibrillation: A new risk factor for contrast-induced nephropathy. Angiology: Sep 27, 2018 (Epub ahead of print).

7. Gruberg L,Mehran R, Dangas G, Mintz GS, Waksman R, Kent KM, Pichard AD, Satler LF, Wu H and Leon MB: Acute renal failure requiring dialysis after percutaneous coronary interventions. Catheter Cardiovasc Interv 52: 409-416, 2001.

8. Bashir AA, Kong V, Skinner D, Bruce J, Laing G, Brysiewicz P and Clarke D: Contrast-induced nephropathy following CT scan for trauma is not rare and is associated with increased mortality in South African trauma patients. Eur J Trauma Emerg Surg: Sep 18, 2018 (Epub ahead of print).

9. Wyatt CM, Camargo M and Coca SG: Prophylactic hydration to prevent contrast-induced nephropathy: Much ado about nothing? Kidney Int 92: 4-6, 2017.

10. Ozkok S and Ozkok A: Contrast-induced acute kidney injury: A review of practical points. World J Nephro 6: 86-99, 2017.

11. Trivedi HS, Moore H, Nasr S, Aggarwal K, Agrawal A, Goel P and Hewett J: A randomized prospective trial to assess the role of saline hydration on the development of contrast nephrotoxicity. Nephron Clin Pract 93: C29-C34, 2003.

12. Tepel M, Aspelin P and Lameire N: Contrast-induced nephropathy: A clinical and evidence-based approach. Circulation 113: 1799-1806, 2006.

13. Ix JH, McCulloch CE and Chertow GM: Theophylline for the prevention of radiocontrast nephropathy: A meta-analysis. Nephrol Dial Transplant 19: 2747-2753, 2004.

14. Bagshaw SM and Ghali WA: Theophylline for prevention of contrast-induced nephropathy: A systematic review and meta-analysis. Arch Intern Med 165: 1087-1093, 2005.

15. Dai B, Liu Y, Fu L, Li Y, Zhang J and Mei C: Effect of theophylline on prevention of contrast-induced acute kidney injury: A meta-analysis of randomized controlled trials. Am J Kidney Dis 60: 360-370, 2012

16. Hu M, Cappeleri J and Lan KK: Applying the law of the iterated logarithm to control type I error in cumulative meta-analysis of binary outcomes. Clin Trials 4: 329-340, 2007.
17. Borm GF and Donders AR: Updating meta-analyses leads to larger type I errors than publication bias. J Clin Epidemiol 62: 825-830.e10, 2009.

18. Brok J, Thorlund K, Gluud C and Wetterslev J: Trial sequential analysis reveals insufficient information size and potentially false positive results in many meta-analyses. J Clin Epidemiol 61: 763-769, 2008.

19. Wetterslev J, Thorlund K, Brok J and Gluud C: Estimating required information size by quantifying diversity in a random-effects meta-analysis. BMC Med Res Methodol 9: 86, 2009.

20. Thorlund K, Devereaux PJ, Wetterslev J, Guyatt G, Ioannidis JP, Thabane L, Gluud LL, Als-Nielsen B and Gluud C: Can trial sequential monitoring boundaries reduce spurious inferences from meta-analyses? Int J Epidemiol 38: 276-286, 2009.

21. Wetterslev J, Thorlund K, Brok J and Gluud C: Trial sequential analysis may establish when firm evidence is reached in cumulative meta-analysis. J Clin Epidemiol 61: 64-75, 2008.

22. Jadad AR, Moore RA, Carroll D, Jenkinson C, Reynolds DJ, Gavaghan DJ and McQuay HJ: Assessing the quality of reports of randomized clinical trials: Is blinding necessary? Control Clin Trials 17: 1-12, 1996.

23. Gandhi MR, Brown P, Romanowski CA, Morcos SK, Campbell S, el Nahas AM and Gray TA: The use of theophylline, an adenosine antagonist in the prevention of contrast media induced nephrotoxicity. Br J Radiol 65: 838, 1992.

24. Abizaid AS, Clark CE, Mintz GS, Dosa S, Popma JJ, Pichard AD, Satler LF, Harvey M, Kent KM and Leon MB: Effects of dopamine and aminophylline on contrast-induced acute renal failure after coronary angioplasty in patients with preexisting renal insufficiency. Am J Cardiol 83: 260-263, A5, 1999.

25. Erley CM, Duda SH, Rehfuss D, Scholtes B, Bock J, Müller $\mathrm{C}$, Osswald $\mathrm{H}$ and Risler T: Prevention of radiocontrast-media-induced nephropathy in patients with pre-existing renal insufficiency by hydration in combination with the adenosine antagonist theophylline. Nephrol Dial Transplant 14: 1146-1149, 1999.

26. Huber W, Ilgmann K, Page M, Hennig M, Schweigart U, Jeschke B, Lutilsky L, Weiss W, Salmhofer H and Classen M: Effect of theophylline on contrast material-nephropathy in patients with chronic renal insufficiency: Controlled, randomized, double-blinded study. Radiology 223: 772-779, 2002.

27. Kapoor A, Kumar S, Gulati S, Gambhir S, Sethi RS and Sinha N: The role of theophylline in contrast-induced nephropathy: A case-control study. Nephrol Dial Transplant 17: 1936-1941, 2002.

28. Huber W, Schipek C, Ilgmann K, Page M, Hennig M, Wacker A, Schweigart U, Lutilsky L, Valina C, Seyfarth M, et al: Effectiveness of theophylline prophylaxis of renal impairment after coronary angiography in patients with chronic renal insufficiency. Am J Cardiol 91: 1157-1162, 2003.

29. Dussol B, Morange S, Loundoun A, Auquier P and Berland Y: A randomized trial of saline hydration to prevent contrast nephropathy in chronic renal failure patients. Nephrol Dial Transplant 21: 2120-2126, 2006.

30. Huber W, Eckel F, Hennig M, Rosenbrock H, Wacker A, Saur D, Sennefelder A, Hennico R, Schenk C, Meining A, et al: Prophylaxis of contrast material-induced nephropathy in patients in intensive care: Acetylcysteine, theophylline, or both? A randomized study. Radiology 239: 793-804, 2006.

31. Demir M, Kutlucan A, Akın H, Aydın O and Sezer MT: Comparison of different agents on radiographic contrast agent induced nephropathy. Eur J Gen Med 5: 222-227, 2008.

32. Baskurt M, Okcun B, Abaci O, Dogan GM, Kilickesmez K, Ozkan AA, Ersanli M and Gurmen T: N-acetylcysteine versus $\mathrm{N}$-acetylcysteine + theophylline for the prevention of contrast nephropathy. Eur J Clin Invest 39: 793-799, 2009.

33. Kinbara T, Hayano T, Ohtani N, Furutani Y, Moritani K and Matsuzaki M: Efficacy of N-acetylcysteine and aminophylline in preventing contrast-induced nephropathy. J Cardiol 55: 174-179, 2010

34. Malhis M, Al-Bitar S and Al-Deen Zaiat K: The role of theophylline in prevention of radiocontrast media-induced nephropathy. Saudi J Kidney Dis Transpl 21: 276-283, 2010.

35. Matejka J, Varvarovsky I, Vojtisek P, Herman A, Rozsival V, Borkova V and Kvasnicka J: Prevention of contrast-induced acute kidney injury by theophylline in elderly patients with chronic kidney disease. Heart Vessels 25: 536-542, 2010.

36. Rohani A: Effectiveness of aminophylline prophylaxis of renal impairment after coronary angiography in patients with chronic renal insufficiency. Indian J Nephrol 20: 80-83, 2010. 
37. Bilasy ME, Oraby MA, Ismail HM and Maklady FA: Effectiveness of theophylline in preventing contrast-induced nephropathy after coronary angiographic procedures. J Intery Cardiol 25: 404-410, 2012.

38. Caglar I, Caglar NT, Conkbayir C, Baskurt M, Aktürk F, Dasli T and Okcun B: Contrast study: Comparision of nephroprotective three protocols: Acetylcysteine-sodium bicarbonate-theophylline, to prevent contrast-induced nephropathy. Russ J Cardiol 105 27-33, 2014.

39. Arabmomeni M, Najafian J, Abdar Esfahani M, Samadi M and Mirbagher L: Comparison between theophylline, $\mathrm{N}$-acetylcysteine, and theophylline plus $\mathrm{N}$-acetylcysteine for the prevention of contrast-induced nephropathy. ARYA Atheroscler 11: 43-49, 2015.

40. Sendeski M, Patzak A and Persson PB: Constriction of the vasa recta, the vessels supplying the area at risk for acute kidney injury, by four different iodinated contrast media, evaluating ionic, nonionic, monomeric and dimeric agents. Invest Radiol 45: $453-457,2010$.
41. Seeliger E, Lenhard DC and Persson PB: Contrast media viscosity versus osmolality in kidney injury: Lessons from animal studies. Biomed Res Int 2014: 358136, 2014.

42. Arend LJ, Bakris GL, Burnett JC Jr, Megerian C and Spielman WS: Role for intrarenal adenosine in the renal hemodynamic response to contrast media. J Lab Clin Med 110: 406-411, 1987.

43. Osswald $\mathrm{H}$ and Schnermann J: Methylxanthines and the kidney. Handb Exp Pharmacol: 391-412, 2011.

44. Weinberger $M$ and Hendeles L: Therapeutic effect and dosing strategies for theophylline in the treatment of chronic asthma. J Allergy Clin Immunol 78: 762-768, 1986.

(i) (9) This work is licensed under a Creative Commons EY No ND Attribution-NonCommercial-NoDerivatives 4.0 International (CC BY-NC-ND 4.0) License. 